\title{
DIAGNÓSTICO DE FALTAS EM TRANSFORMADORES DE POTÊNCIA ATRAVÉS DO MÉTODO DE SEGMENTAÇÕES GEOMÉTRICAS SUCESSIVAS
}

\author{
Wander Antunes Gaspar Valente*, Edimar José de Oliveira*, Leonardo de Mello \\ Honório*, Lucas CorrêA NetTo MaChado* \\ * Programa de Pós-Graduação em Engenharia Elétrica, Faculdade de Engenharia, \\ Universidade Federal de Juiz de Fora, MG, Brasil
}

Emails: wandergaspar@ieee.org, edimar.oliveira@ufjf.edu.br, leonardo.honorio@ufjf.edu.br, lucas.correa@engenharia.ufjf.br

\begin{abstract}
This paper uses a new method of pattern recognition to diagnose incipient faults in transformers using as input information of the gases dissolved in insulating oil. The method allows the construction and training of a neural network using a geometrical approach to the problem. The implemented algorithm was tested using data sets available in the literature and the results of tests carried out indicate high levels of accuracy and computational performance.
\end{abstract}

Keywords - Neural networks, Pattern recognition, Power transformers, Incipient faults.

Resumo - Este trabalho apresenta um novo método classificador para o diagnóstico de faltas incipientes em transformadores de potência com base em levantamento de dados pela técnica de análise de gases dissolvidos. O classificador fundamenta-se na construção e treinamento de redes neurais artificiais a partir de segmentações geométricas sucessivas. Essa abordagem permite gerar tanto a topologia quanto o peso de cada neurônio sem a necessidade da especificação dos parâmetros da rede. O classificador de padrões foi testado utilizando-se conjuntos de dados de faltas em transformadores de potência disponíveis na literatura e os resultados dos testes realizados indicam altas taxas de acerto.

Palavras-chave - Redes neurais, Reconhecimento de padrões, Transformadores de potência, Faltas incipientes.

\section{Introdução}

Transformadores de potência são considerados como itens de custo elevado em Sistemas Elétricos de Potência (SEP). Faltas em transformadores podem resultar em interrupções da transmissão de energia com graves consequências. Estas características conduzem à necessidade de cuidadoso monitoramento da operação do equipamento ao longo de toda a vida útil. O correto diagnóstico de faltas em transformadores representa um quesito importante não apenas para a segurança do próprio dispositivo mas também para a confiabilidade do SEP como um todo (Ganyun et al., 2005).

No âmbito das concessionárias de energia elétrica pode-se relatar uma série de modernas técnicas de diagnóstico capazes de avaliar a condição de isolamento de transformadores de potência. Entre os métodos que possuem maior aceitação, pode-se análise de umidade no óleo isolante, a análise de gases dissolvidos, a medição do grau de polimerização e a análise de furano por cromatografia líquida de alta eficiência (Saha, 2003).

Nesse contexto, a análise de gases dissolvidos (DGA, do inglês Dissolved Gas Analysis) inclui-se entre os métodos citados que se destacam na literatura científica (Wang, 2003). Na ocorrência de uma falta interna no equipamento, as taxas de degradação do óleo e dos materiais isolantes sólidos, tais como papel e cartão prensados fabricados a partir de celulose são significativamente aumentados (Saha, 2003).
Os gases mais importantes produzidos ao longo da operação de um transformador de potência são o hidrogênio $\left(H_{2}\right)$ e os hidrocarbonetos metano $\left(\mathrm{CH}_{4}\right)$, etano $\left(\mathrm{C}_{2} \mathrm{H}_{6}\right)$, etileno $\left(\mathrm{C}_{2} \mathrm{H}_{4}\right)$, acetileno $\left(\mathrm{C}_{2} \mathrm{H}_{2}\right)$; monóxido de carbono $(\mathrm{CO})$ e dióxido de carbono $\left(\mathrm{CO}_{2}\right)$; outros gases não relacionados à faltas, como nitrogênio $\left(N_{2}\right)$ e oxigênio $\left(\mathrm{O}_{2}\right)$.

O presente trabalho utiliza a classificação de faltas proposta em (IEEE Guide for the Interpretation of Gases Generated in Oil-Immersed Transformers, 2009), cujas categorias são (a) estado normal, (b) sobreaquecimento, (c) falta elétrica - descarga de baixa intensidade e (d) falta elétrica - descarga de alta intensidade. A metodologia proposta utiliza duas bases de dados distintas para validação para as quais considera os valores medidos referentes aos gases hidrogênio, metano, etano, etileno e acetileno para fins de diagnóstico de faltas.

A série de dados utilizada para a validação da abordagem utilizada foi obtida a partir do relatório 60599 e tabela TB10 do IEC, conforme apresentado em Duval and dePabla (2001). Esta base de dados contém um total de 130 dados amostrais, onde 26 amostras são identificadas por inspeção como descarga de baixa energia, 48 amostras identificadas como descarga de alta energia, 18 amostras de sobreaquecimento e 38 amostras de estado normal. 


\section{Método de Segmentações Geométricas Sucessivas}

Problemas de classificação ocorrem em Engenharia Elétrica bem como em diversas outras áreas de pesquisa (Abe, 2010). As abordagens mais utilizadas atualmente consistem em técnicas de classificação geométricas - como ênfase em máquinas de vetores de suporte (SVM, do inglês Support Vector Machines) — e em teorias oriundas da área de inteligência computacional, com destaque para o paradigma conexionista, representado pelos trabalhos com redes neurais artificiais (RNA) (Ogiela and Jain, 2012).

O reconhecimento de padrões no contexto de problemas multi-classe implica na obtenção de um sistema capaz de mapear com precisão um espaço de entrada para um espaço de saída constituído por duas ou mais classes de padrões $(\mathrm{Ou}$ and Murphey, 2007). O diagnóstico de faltas em transformadores de potência com base na classificação proposta em (IEEE Guide for the Interpretation of Gases Generated in Oil-Immersed Transformers, 2009) consiste em um problema multiclasse, cujas classes são representadas pelas categorias normal, sobreaquecimento, descarga de baixa intensidade e descarga de alta intensidade.

A abordagem mais utilizada para classificação de padrões multiclasse consiste em decompor o problema em vários subproblemas compostos por duas classes apenas. Encontra-se na literatura diversas abordagens para processar a decomposição de padrões multiclasse, tanto para algoritmos baseados em SVM (Hsu and Lin, 2002) quanto para RNA (Anand et al., 1995).

\subsection{Descrição do Método Empregado}

O algoritmo classificador de padrões utilizado neste trabalho fundamenta-se em uma nova técnica denominada Método de Segmentações Geométricas Sucessivas (MSGS) para a geração automática de uma RNA. Especificamente, o MSGS emprega técnicas de segmentação geométrica para determinar tanto a topologia da rede quanto o peso de cada neurônio sem a necessidade de especificação de parâmetros. Em razão desta característica, o método introduzido reveste-se de relevância ao contribuir para o equacionamento da etapa de treinamento da rede, uma das principais dificuldades imputadas ao emprego de RNAs em problemas complexos (Wilamowski, 2010).

Abordagens baseadas em interpretações geométricas no contexto de RNAs são identificadas em diversos trabalhos na literatura (Chen et al., 1997), (Gentile and Sznaier, 2001). O MSGS fundamenta-se na representação do neurônio através de um hiperplano associado a uma função de ativação. No contexto de um classificador, o objetivo consiste em determinar um conjunto de hiperplanos que permita separar as diversas classes de dados em estudo.

Inicialmente, cada classe é modelada através de um envelope. Caso os envelopes sejam linearmente separáveis, pode-se definir um ou mais hiperplanos de separação. Os parâmetros do hiperplano permitem determinar os pesos do neurônio capaz de representar a separação entre as classes em estudo. Caso os envelopes não sejam linearmente separáveis, os dados são subdivididos, gerando-se novos envelopes menores e mais precisos para a representação das classes. Este processo é repetido até que seja detectada a separação entre os envelopes ou um critério de parada seja alcançado.

Os neurônios de uma RNA podem ser definidos a partir de uma interpretação geométrica (Bose and Garga, 1993). Matematicamente, é possível expressar esta característica a partir da definição de hiperplano $\mathcal{X}$ como um conjunto de pontos $\vec{x} \in \mathbb{R}^{n}$ que satisfaz a expressão:

$$
\sum_{i=1}^{n} x^{i} w^{i}+b=0
$$

onde $\vec{w} \in \mathbb{R}^{n}$ é um vetor de pesos e $b$ é um escalar. Pode-se afirmar que $\mathcal{X}$ divide o espaço $\mathbb{R}^{n}$ em um subespaço positivo $\mathcal{X}^{+}: \sum_{i=1}^{n}=x_{i} w_{i}+b \geq 0 \mathrm{e}$ um subespaço negativo $\mathcal{X}^{-}: \sum_{i=1}^{n}=x_{i} w_{i}+b<0$ (Gentile and Sznaier, 2001).

Com base na equação (1) e tomando-se como referência duas classes de dados no espaço $\mathbb{R}^{n}$ representadas pelos conjuntos de pontos $\vec{x}^{1}$ e $\vec{x}^{2}$, então, se $x^{1} \neq x^{2}$, têm-se que:

$$
\operatorname{sgn}\left(\sum_{i=1}^{n} x_{i}^{1} w_{i}+b\right) \neq \operatorname{sgn}\left(\sum_{i=1}^{n} x_{i}^{2} w_{i}+b\right)
$$

onde para $z \in \mathbb{R}$ :

$$
\operatorname{sgn}(z)=\left\{\begin{aligned}
+1, & \text { se } z>0 \\
-1, & \text { se } z<0 \\
0, & \text { se } z=0
\end{aligned}\right.
$$

A equação (2) afirma que existe pelo menos um vetor de pesos $\vec{w}$ e um escalar $b$ que se aplicados a $\vec{x}^{1}$ e $\vec{x}^{2}$ irão produzir sinais diferentes na saída. Conclui-se, portanto, que se devidamente ajustados, $\vec{w}$ e $b$ irão definir um hiperplano de separação $\mathcal{X}$ entre as classes de dados representadas por $\vec{x}^{1}$ e $\vec{x}^{2}$.

Observa-se que $\vec{w}$ e $b$ são os pesos atribuídos a uma entrada de um neurônio com função de ativação binária dada por (3). Partindo-se da definição do hiperplano de separação, conforme equação (1), pode-se inferir que um conjunto de neurônios com os respectivos pesos devidamente ajustados pode gerar uma saída capaz de identificar um conjunto de pontos em um subespaço de $\mathbb{R}^{n}$.

Nesse contexto, o método MSGS visa delimitar as classes de dados em subespaços representados por envelopes — descritos na Seção 2.1.1 - 
e, a partir dessas estruturas, definir os hiperplanos de separação adequados à geração da RNA que permita a correta classificação das instâncias fornecidas como entrada.

\subsubsection{Representação por Envelopes}

A primeira etapa do MSGS consiste na representação dos dados por um envelope. Podem ser encontradas na literatura diversas topologias baseadas em volumes geométricos convexos. Gregory et al. (1999) apontam a Oriented Bounded Box (OBB) como uma alternativa eficiente. OBB define um envelope na forma de um paralelepípedo retangular alinhado ao eixo de maior distribuição dos pontos. Este eixo é obtido a partir do cálculo dos autovalores e autovetores da matriz de covariância das instâncias que compõem a classe. Observa-se que o método OBB foi desenvolvido para o espaço tridimensional. Uma vez que o presente trabalho busca classificar classes $n$-dimensionais, a metodologia adotada foi adaptada para espaços de instâncias de múltiplas dimensões e recebeu a denominação de Oriented Bounded HyperBox (OBHB).

Para efeito de ilustração, a Figura 1 apresenta os autovetores $\pi_{1}$ e $\pi_{2}$ calculados para um conjunto de pontos $X$ no espaço $\mathbb{R}^{2}$ a partir da matriz de covariância. Os autovetores alinham-se com a máxima dispersão de $X$ e os correspondentes autovalores representam a magnitude da dispersão. $X_{0}$ identifica o ponto médio de $X$.

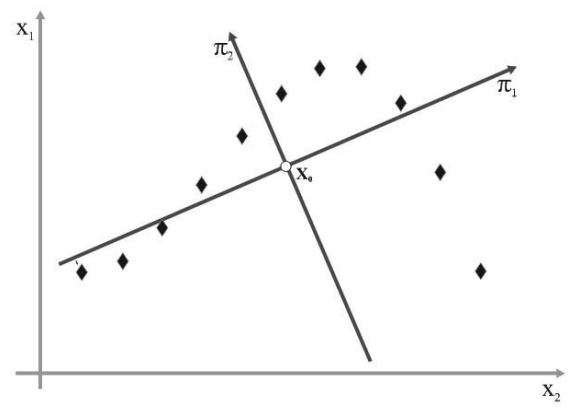

Figura 1: Autovetores calculados a partir da matriz de covariância

O ponto $X_{0}$ e os eixos ortogonais alinhados com os autovetores permitem definir um novo sistema de coordenadas sobre o qual são calculadas as projeções de $X$ (Figura 2). A partir dos valores máximos e mínimos das projeções $\operatorname{Pr}(X, \pi)$ é possível definir uma OBB $\mathcal{H}$ capaz de envolver o conjunto de pontos $X$ (Figura 3 ).

Pode-se observar ainda na Figura 3 que o centro geométrico da $\mathrm{OBB}$, representado por $\mathcal{H}_{0}$, não coincide com $X_{0}$, ponto médio de $X$. Também encontram-se identificados na Figura 3 as projeções parciais de $\mathcal{H}$ sobre cada um dos eixos $\pi$, representados por $\delta(\mathcal{H}, \pi)$. Como será apresentado na Seção ??, as projeções parciais $\delta(\mathcal{H}, \pi)$ e também as projeções totais $\Delta(\mathcal{H}, \pi)=2 \delta(\mathcal{H}, \pi)$ serão

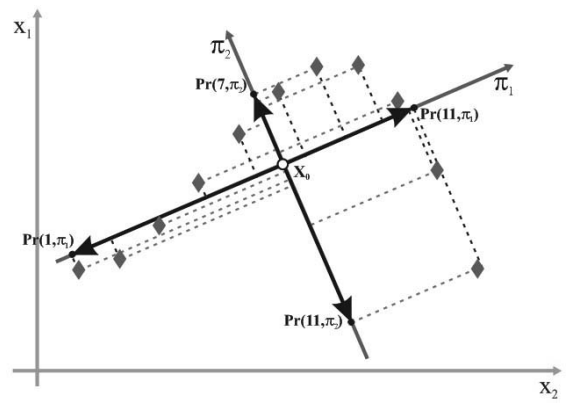

Figura 2: Projeções $\operatorname{Pr}(X, \pi)$

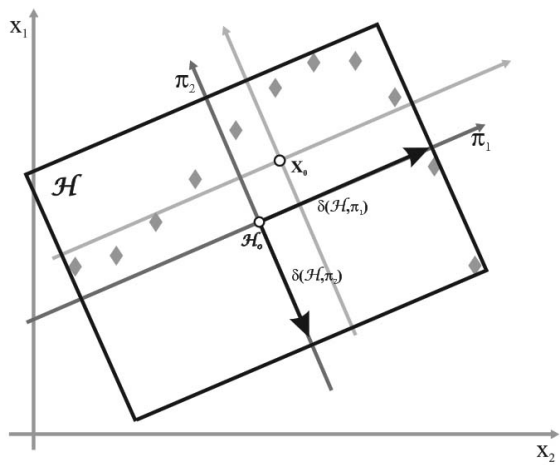

Figura 3: OBB envolvente de $X$

utilizadas na etapa de teste de colisão entre OBBs.

A segunda etapa do MSGS consiste em identificar se os dois envelopes $\mathcal{H}_{1}$ e $\mathcal{H}_{2}$ estão em colisão. O teste de colisão adotado no MSGS baseia-se no Teorema do Eixo de Separação (TES), desenvolvido por Gottschalk (2000) para aplicação em classes de dados contendo instâncias no espaço $\mathbb{R}^{3}$.

Com base no TES, pode-se afirmar que duas OBBs não estão em colisão se existir pelo menos um eixo sobre o qual a projeção dos envelopes não é contínua. A Figura 4 ilustra este conceito, onde $\left(\pi_{1}, \pi_{2}\right)$ e $\left(\pi_{3}, \pi_{4}\right)$ representam, respectivamente, as bases de dois sistemas de coordenadas que contêm duas OBBs, $\mathcal{H}^{1}$ e $\mathcal{H}^{2}$. Ainda na Figura 4, $\Delta_{\mathcal{H}_{0}^{1}, \mathcal{H}_{0}^{2}, 3}$ representa um vetor alinhado com o eixo $\pi_{3}=x_{2}$ que estende-se a partir dos centros $\mathcal{H}_{0}^{1} \mathrm{e}$ $\mathcal{H}_{0}^{2}$ das respectivas OBBs. As projeções de $\mathrm{H}^{1}$ sobre os eixos $\pi_{1}$ e $\pi_{2}$ e as projeções de $\mathrm{H}^{2}$ sobre os eixos $\pi_{3}$ e $\pi_{4}$ são representadas, respectivamente, por $\delta_{1,1}, \delta_{1,2}, \delta_{2,3}$ e $\delta_{2,4}$.

Por fim, considerando-se mais uma vez a Figura 4 , ao aplicar-se o TES sobre o eixo $\pi_{3}=$ $x_{2}$, é possível observar graficamente que $\left|\delta_{\mathcal{H}^{1}, 3}\right|+$ $\left|\delta_{\mathcal{H}^{2}, 3}\right|<\left|\Delta_{\mathcal{H}_{0}^{1}, \mathcal{H}_{0}^{2}, 3}\right|$. Em consequência, pode-se concluir que o eixo $\pi_{3}$ constitui um eixo de separação entre $\mathcal{H}^{1}$ e $\mathcal{H}^{2}$. Graficamente, observa-se ainda que $\pi_{1}$ constitui um segundo eixo de separação entre $\mathcal{H}^{1}$ e $\mathcal{H}^{2}$, ao contrário dos eixos $\pi_{2}$ e $\pi_{4}$.

A aplicação do MSGS requer a identificação de todos os eixos de separação entre as OBHBs consideradas no problema em estudo. Este requisito justifica-se em razão da abordagem geomé- 


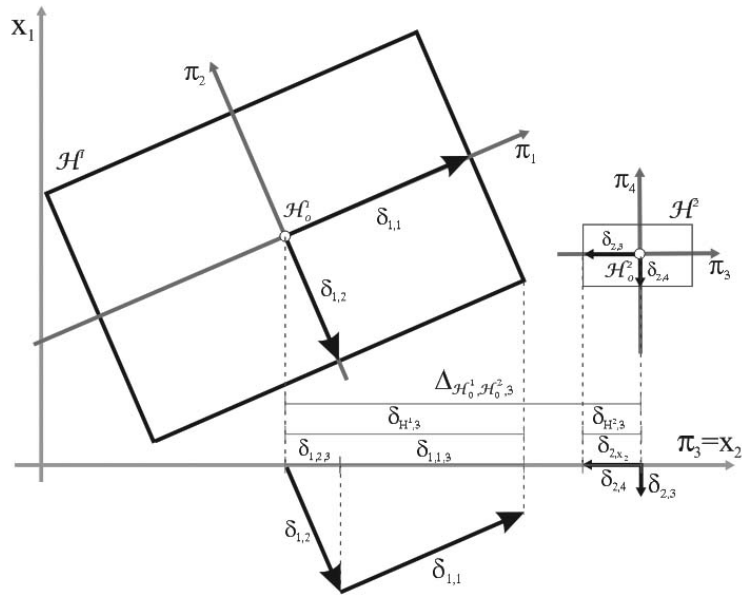

Figura 4: Aplicação do TES no espaço $\mathbb{R}^{2}$

trica adotada para a construção do classificador. A RNA requer a definição de hiperplanos de separação normais a todos os eixos de separação encontrados para as classes de dados em estudo. Os hiperplanos de separação serão responsáveis pela especificação das funções de ativação capazes de inferir a classificação das instâncias de dados.

Por conseguinte, uma das contribuições do MSGS consiste em estender o TES para classes de dados com instâncias em $\mathbb{R}^{n}$ a partir do Teorema do Hiperplano de Separação (THS). Sejam duas OBHBs $\mathcal{H}_{i}$ e $\mathcal{H}_{j}$ pertencentes a $\mathbb{R}^{n}$. Se as OBHBs não estiverem em colisão, então existe um eixo de separação $\pi_{p}$ que contém as projeções $\delta_{\mathcal{H}^{i}, p}$ e $\delta_{\mathcal{H}^{j}, p}$. É possível então definir-se um segmento de reta $\mathcal{S}$ sobre $\pi_{p}$ tal que

$$
|\mathcal{S}|=\left|\Delta_{\mathcal{H}_{0}^{i}, \mathcal{H}_{0}^{j}, p}\right|-\left|\delta_{\mathcal{H}^{i}, p}\right|-\left|\delta_{\mathcal{H}^{j}, p}\right| .
$$

From the line segment $\mathcal{S}$, it is possible to define a hyperplane $\mathcal{X} \in \mathbb{R}^{n}$ normal to the axis $\pi_{p}$ and containing a point $x^{r} \subset \mathcal{S}$. Hence $\mathcal{X}\left(\mathcal{H}^{i}, \mathcal{H}^{j}\right)$ represents a separating hyperplane between $\mathcal{H}^{i}$ and $\mathcal{H}^{j}$.

Figure 5 shows a separating hyperplane $\mathcal{X}\left(\mathcal{H}^{1}, \mathcal{H}^{2}\right)$ orthogonal to the axis $\pi_{3}$ at the point $x^{r} \in \mathcal{S}$.

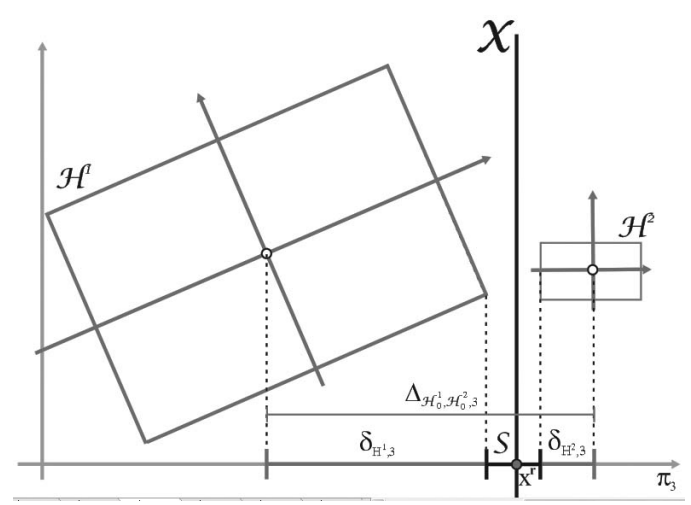

Figura 5: Separating hyperplane $\mathcal{X}$

É possível ainda observar através da Figura 2 a existência de infinitos hiperplanos de separação entre $\mathcal{H}^{1}$ e $\mathcal{H}^{2}$ uma vez que qualquer ponto $x \subset \mathcal{S}$ é candidato para a construção de $\mathcal{X}\left(\mathcal{H}^{1}, \mathcal{H}^{2}\right)$. Nesse trabalho considera-se $x^{r}$ como o ponto médio de $\mathcal{S}$.

Uma colisão entre OBHBs significa que não é possível identificar um hiperplano de separação entre as classes. Nesta situação, torna-se necessário aumentar a representatividade dos envelopes a partir dos dados em estudo.

O tratamento de colisão no MSGS baseia-se em uma técnica de segmentação geométrica proposta por Gottschalk et al. (1996). Esta abordagem, denominada árvore de colisão, propõe a divisão dos dados de uma classe através de uma hierarquia de OBHBs. O propósito deste processo consiste em obter, a cada novo subnível, envelopes que representem com maior precisão as instâncias de dados.

A heurística adotada pelo MSGS para a divisão de uma OBHB $\mathcal{H}_{n}$ em duas novas OBHBs menores, $\mathcal{H}_{n 1}$ e $\mathcal{H}_{n 2}$, fundamenta-se na separação das projeções de valores positivos e negativos em relação ao eixo $\pi_{p}$ relacionado ao maior autovalor. Esta heurística tem por objetivo dividir o conjunto de pontos no sentido do maior eixo de dispersão.

A Figura 6 ilustra a abordagem empregada para a divisão de uma OBHB em $\mathbb{R}^{2}$. O eixo $\pi_{1}$ possui o maior autovalor associado. Então, de acordo com a heurística, $\mathcal{H}_{1}$ divide-se em $X_{0}$ e gera os envelopes do subnível seguinte, $\mathcal{H}_{1,1}$ e $\mathcal{H}_{1,2}$.

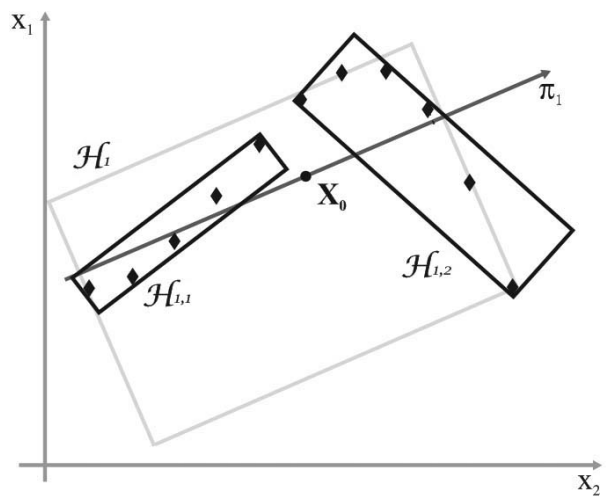

Figura 6: Divisão de uma OBHB em dois envelopes menores, $\mathcal{H}_{1,1}$ e $\mathcal{H}_{1,2}$

\subsection{Implementação da RNA}

Um neurônio pode ser interpretado como um hiperplano $\mathcal{X}$ que divide o espaço $\mathbb{R}^{n}$ em dois subespaços $\mathcal{X}^{+}$e $\mathcal{X}^{-}$. Portanto, no contexto do MSGS, cada hiperplano gerado conduz à especificação dos parâmetros de um dos neurônios da rede.

A RNA gerada pela metodologia empregada possui tipologia do tipo feedforward e contém duas camadas intermediárias. A primeira camada é 
composta pelos neurônios associados aos hiperplanos. A segunda camada tem o objetivo de indicar a região do espaço $n$-dimensional onde se encontra cada um dos dados de entrada fornecidos para a estrutura. A Figura 7 exibe o modelo de rede neural construída com base no MSGS.

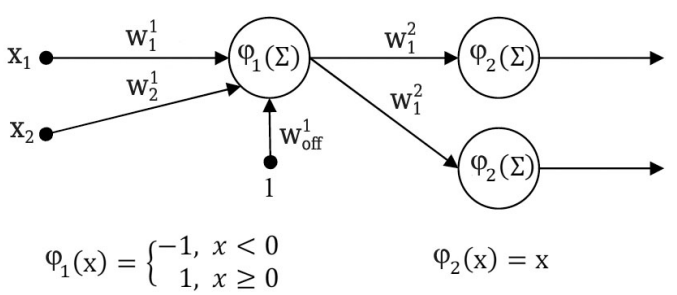

Figura 7: Modelo de RNA com base no MSGS

Os valores dos pesos da primeira camada oculta, representados por $w_{i}^{1}$, referem-se aos vetores normais aos hiperplanos gerados. Os valores de offset $w_{\text {off }}^{1}$ são obtidos através do produto escalar dos pontos médios dos hiperplanos e vetores normais. Pode-se observar ainda pela Figura 7, a especificação das funções de ativação $\varphi_{1}$ e $\varphi_{2}$ respectivamente para a primeira e segunda camadas da RNA.

Os valores dos pesos $w_{i}^{2}$ referentes à segunda camada oculta representam os padrões que determinam cada uma das regiões definidas pelos hiperplanos gerados. Os valores de $w_{i}^{2}$ podem ser positivos e negativos e a magnitude é inversamente proporcional á quantidade de entradas da camada. Por exemplo, na hipótese de duas entradas para a segunda camada, os valores possíveis para $w_{i}^{2}$ seriam $(-1,+1) \cdot \frac{1}{2}=(-0.5,+0.5)$. Esta abordagem conduz a uma saída igual +1 quando um dado de entrada estiver contido em uma determinada região do espaço.

Para exemplificar o processo de implementação e emprego de uma RNA gerada pelo MSGS, tome-se como dados de entrada um conjunto de instâncias referentes a duas classes no espaço $\mathbb{R}^{2}$, representadas por círculos e losangos. A Figura 8 exibe, além das instâncias, as OBHBs e os hiperplanos de separação gerados segundo a metodologia apresenta neste trabalho.

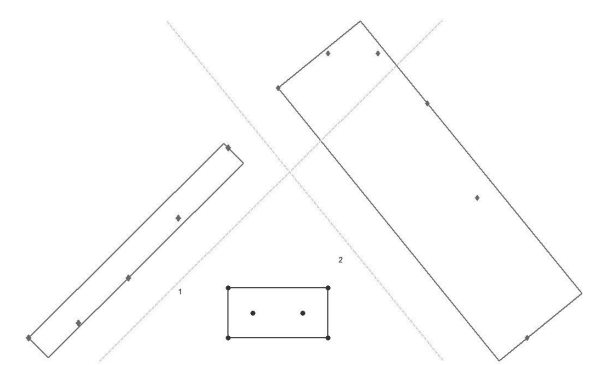

Figura 8: OBHBs e hiperplanos de separação gerados pelo MSGS

A Tabela 1 apresenta os valores obtidos tanto para os pesos $w_{i}^{1}$ quanto para os pesos $w_{\text {off }}^{1}$, ob- tidos a partir dos pontos médios $x_{0}$, para os hiperplanos de separação, conforme observados na Figura 8.

Tabela 1: Hiperplanos de separação gerados pelo MSGS

\begin{tabular}{c|cc|cc}
\hline & \multicolumn{2}{|c|}{ Hiperplano 1 } & \multicolumn{2}{c}{ Hiperplano 2 } \\
\hline$w_{i}^{1}$ & 0.7048 & -0.7094 & -0.6160 & -0.7757 \\
$x_{0}$ & 0.2897 & 0.0997 & 0.6541 & 0.1720 \\
$w_{\text {off }}^{1}$ & \multicolumn{2}{|c|}{0.1334} & \multicolumn{2}{c}{-0.6160} \\
\hline
\end{tabular}

\section{Diagnóstico de Faltas}

O escopo deste trabalho inclui o diagnóstico de faltas em transformadores de potência tomando-se como parâmetros de entrada bases de dados disponíveis na literatura sobre análise de gases dissolvidos (DCA). O algoritmo classificador deve diagnosticar quatro categorias de faltas no equipamento: (a) estado normal, (b) sobreaquecimento, (c) falta elétrica - descarga de baixa intensidade e (d) falta elétrica - descarga de alta intensidade. $\mathrm{O}$ algoritmo classificador foi desenvolvido a partir do método MSGS apresentado na Seção 2.1. A implementação do software foi escrita em Matlab versão R2010b em ambiente operacional Windows 7 sob hardware composto por processador Intel Core i7 $2.93 \mathrm{GHz}$ e $8 \mathrm{~Gb}$ de memória RAM.

Para validação do classificador baseado no método MSGS, os resultados encontrados foram comparados tanto com a toolbox de redes neurais quanto de bio-informática do Matlab versão R2010b. Especificamente no toolbox de redes neurais, utilizou-se a função patternnet para reconhecimento de padrões através de uma RNA feedforward com a especificação de 30 neurônios para a camada oculta. Para a implementação do classificador SVM, foram empregadas as funções svmtrain e svmclassify do toolbox de bioinformática.

Tabela 2: Diagnóstico de faltas em transformadores para dados segundo Duval and dePabla (2001)

\begin{tabular}{lcccc}
\hline Etapa & Instâ. & MSGS & RNA & SVM \\
\hline \hline Normal & 10 & $\mathbf{1 0}$ & 7 & 1 \\
\hline Falta & 32 & 29 & 31 & $\mathbf{3 2}$ \\
\hline \hline Descarga & 22 & 19 & $\mathbf{2 0}$ & $\mathbf{2 0}$ \\
\hline Sobreaquec. & 10 & $\mathbf{8}$ & 6 & 6 \\
\hline \hline Baixa Intens. & 10 & $\mathbf{8}$ & 6 & 7 \\
\hline Alta Intens. & 12 & $\mathbf{1 2}$ & 9 & 9 \\
\hline
\end{tabular}

Conforme pode ser observado a partir da Tabela 2, o método MSGS apresenta os melhores resultados para a maioria das classes. Mesmo nos casos em que um dos demais métodos obtém valores mais satisfatórios, o MSGS conduz a altas taxas de acerto para as instâncias avaliadas. 


\section{Conclusões}

Foi apresentado um novo método classificador para o diagnóstico de faltas em transformadores de potência. O método fundamenta-se na construção e treinamento de RNAs a partir de segmentações geométricas sucessivas. Essa abordagem permite gerar tanto a topologia quanto o peso dos neurônios sem a especificação dos parâmetros da rede. O classificador foi testado utilizando-se conjuntos de dados disponíveis na literatura e os resultados dos testes realizados foram promissores, apresentando altas taxas de acerto.

\section{Agradecimentos}

Os autores agradecem o apoio dado pela CAPES, CNPq, FAPEMIG e INERGE.

\section{Referências}

Abe, S. (2010). Support Vector Machines for Pattern Classification (Advances in Pattern Recognition), 2nd edn, Springer-Verlag, Secau-cus, NJ, USA. DOI: 10.1007/978-1-84996-098-4

Anand, R., Mehrotra, K., Mohan, C. and Ranka, S. (1995). Efficient classification for multiclass problems using modular neural networks, Neural Networks, IEEE Transactions on 6(1): 117-124.

Bose, N. and Garga, A. (1993). Neural network design using voronoi diagrams, Neural Networks, IEEE Transactions on 4(5): 778-787.

Chen, Y. Q., Damper, R. and Nixon, M. (1997). On neural-network implementations of $\mathrm{k}$ nearest neighbor pattern classifiers, Circuits and Systems I: Fundamental Theory and Applications, IEEE Transactions on 44(7): 622629.

Duval, M. and dePabla, A. (2001). Interpretation of gas-in-oil analysis using new iec publication 60599 and iec tc10 databases, Electrical Insulation Magazine, IEEE 17(2): 31-41. DOI: $10.1109 / 57.917529$

Ganyun, L., Haozhong, C., Haibao, Z. and Lixin, D. (2005). Fault diagnosis of power transformer based on multi-layer svm classifier, Electric Power Systems Research 74(1): 1-7. DOI: $10.1016 /$ j.epsr.2004.07.008

Gentile, C. and Sznaier, M. (2001). An improved voronoi-diagram-based neural net for pattern classification, Neural Networks, IEEE Transactions on 12(5): 1227-1234.

Gottschalk, S. (2000). Collision queries using oriented bounding boxes, $\mathrm{PhD}$ thesis, The University of North Carolina, Chapel Hill - NC - USA.
Gottschalk, S., Lin, M. C. and Manocha, D. (1996). Obbtree: a hierarchical structure for rapid interference detection, Proceedings of the 23rd annual conference on Computer graphics and interactive techniques, SIGGRAPH '96, ACM, New York, NY, USA, pp. $171-180$.

Gregory, A., Lin, M., Gottschalk, S. and Taylor, R. (1999). A framework for fast and accurate collision detection for haptic interaction, Virtual Reality, 1999. Proceedings., IEEE, pp. 38-45.

Hsu, C.-W. and Lin, C.-J. (2002). A comparison of methods for multiclass support vector machines, Neural Networks, IEEE Transactions on 13(2): 415-425.

IEEE Guide for the Interpretation of Gases Generated in Oil-Immersed Transformers (2009). IEEE Std C57.104-2008 (Revision of IEEE Std C5\%.104-1991) pp. C1-27.

Ogiela, M. R. and Jain, L. C. (2012). Recent advances in pattern classification, in $\mathrm{M}$. R. Ogiela and L. C. Jain (eds), Computational Intelligence Paradigms in Advanced Pattern Classification, Vol. 386 of Studies in Computational Intelligence, Springer Berlin Heidelberg, pp. 1-4.

Ou, G. and Murphey, Y. L. (2007). Multi-class pattern classification using neural networks, Pattern Recogn. 40(1): 4-18. DOI: 10.1016/j.patcog.2006.04.041

Saha, T. K. (2003). Review of modern diagnostic techniques for assessing insulation condition in aged transformers, Dielectrics and Electrical Insulation, IEEE Transactions on 10(5): 903-917.

Wang, M.-H. (2003). A novel extension method for transformer fault diagnosis, Power Delivery, IEEE Transactions on 18(1): 164-169.

Wilamowski, B. (2010). Challenges in applications of computational intelligence in industrial electronics, Industrial Electronics (ISIE), 2010 IEEE International Symposium on, pp. 15-22. 